\title{
Sanmiao formula inhibits chondrocyte apoptosis and cartilage matrix degradation in a rat model of osteoarthritis
}

\author{
YING XU $^{1 *}$, GUO-JING DAI ${ }^{1 *}$, QIAN LIU ${ }^{1}$, ZHEN-LI LIU ${ }^{2}$, ZHI-QIAN SONG ${ }^{2}$, \\ $\mathrm{LI} \mathrm{LI}^{3}$, WEI-HENG CHEN ${ }^{3}$ and NA LIN ${ }^{1}$ \\ Institutes of ${ }^{1}$ Chinese Materia Medica and ${ }^{2}$ Basic Theory, China Academy of Chinese Medical Sciences, Beijing 100700; \\ ${ }^{3}$ Wangjing Hospital, China Academy of Chinese Medical Sciences, Beijing 100102, P.R. China
}

Received February 5, 2014; Accepted July 14, 2014

DOI: $10.3892 /$ etm.2014.1862

\begin{abstract}
Sanmiao formula (SM) is a basic prescription for the treatment of gouty and rheumatoid arthritis that has been used in China over a long period of history. However, there is no evidence associating SM with the treatment of osteoarthritis (OA). In this study, a characterization of the anti-OA effect of SM was conducted using an in vivo rat model induced by anterior cruciate ligament transection and medial meniscus resection (ACLT plus MMx), together with in vitro studies using chondrocytes for further molecular characterization. Rats subjected to ACLT plus MMx were treated with SM at doses of $0.63,1.25$ and $2.5 \mathrm{~g} / \mathrm{kg}$ per day for three or six weeks. SM treatment significantly inhibited the histopathological changes of articular cartilage damage and synovial inflammation in the rats following ACLT plus MMx. SM $(2.5 \mathrm{~g} / \mathrm{kg})$ clearly inhibited chondrocyte apoptosis and prevented cartilage matrix degradation, which was indicated by the increased proteoglycan and collagen content, particularly with regard to type II collagen expression in articular cartilage. Furthermore, SM $(2.5 \mathrm{~g} / \mathrm{kg})$ markedly inhibited the release of interleukin (IL)-1 $\beta$, tumor necrosis factor- $\alpha$ and nitric oxide in serum, while simultaneously increasing the levels of bone morphogenetic protein- 2 and transforming growth factor- $\beta$ in the circulation. Notably, SM $(2.5 \mathrm{~g} / \mathrm{kg})$ clearly attenuated the OA-augmented expression of matrix metalloproteinase (MMP)-13 and augmented the OA-reduced expression of tissue inhibitor of metalloproteinase (TIMP)-1 in the knee joints. In addition, SM significantly reduced the proportion of early and late apoptotic
\end{abstract}

Correspondence to: Professor Na Lin, Institute of Chinese Materia Medica, China Academy of Chinese Medical Sciences, 16 Dongzhimen Nanxiao Road, Dongcheng, Beijing 100700, P.R. China

E-mail: linna888@163.com

*Contributed equally

Key words: Sanmiao formula, chondrocytes, proteoglycan, collagen, inflammatory cytokines, metalloproteinases, tissue inhibitors of metalloproteinase and sub- $\mathrm{G}_{1}$ phase cells, and clearly decreased the expression of MMP-13 and increased that of TIMP-1 at the mRNA and protein levels in IL-1 $\beta$-induced chondrocytes. These findings provide the first evidence that SM effectively treats OA by inhibiting chondrocyte apoptosis, cartilage matrix degradation and the inflammatory response.

\section{Introduction}

Osteoarthritis (OA) is a degenerative joint disease characterized by the loss of chondrocyte function and extracellular matrix (ECM) destruction (1). The stability of articular cartilage depends on the biosynthetic activities of chondrocytes, which synthesize appropriate ECM molecules to maintain cartilage homeostasis $(2,3)$. Chondrocyte apoptosis alters the cartilage matrix synthesis, leading to matrix degeneration and destruction, and finally to OA (4). Cartilage ECM molecules including type II collagen and sulfated proteoglycan play a crucial role in regulating chondrocyte functions by facilitating cell-matrix interactions (5). The development and progression processes of OA are now considered to involve inflammation (6). Interleukin (IL)-1 $\beta$, tumor necrosis factor (TNF)- $\alpha$ and nitric oxide (NO) are produced by activated synoviocytes, mononuclear cells or chondrocytes, and have been reported to induce apoptosis in chondrocytes and ECM degradation $(7,8)$. In response to IL-1 $\beta$, chondrocytes secrete matrix metalloproteinases (MMPs), which induce chondrocyte apoptosis and inhibit ECM biosynthesis $(9,10)$. Additionally, the activity of MMPs is also regulated by the inhibition of endogenous tissue inhibitors of metalloproteinase (TIMPs), and the imbalance in the ratio of TIMPs and MMPs results in continued destruction in OA (11).

Sanmiao formula (SM) is a compound prescription that comprises three ingredients: Chinese Phellodendri Cortex, Atractylodis Rhizoma and Achyranthis Bidentatae Radix, in a specified ratio (12). SM has been used in traditional Chinese medicine since the Ming Dynasty (15th century common era) due to its function of clearing heat and removing dampness. Currently SM and modified SM are commonly used clinically for the treatments of gouty and rheumatoid arthritis $(13,14)$. However, the effects of SM on OA remain uncharacterized. In the present study, the effects of SM on articular cartilage damage were investigated, with a focus on chondrocyte 
apoptosis and cartilage matrix degradation, as well as the inflammatory response, using a rat model of OA.

\section{Materials and methods}

Herbal preparation. SM was prepared as described in the Chinese Pharmacopeia of 2010 (12). Atractylodis rhizome (600 g, baked with salt), Phellodendri Chinese Cortex (400 g) and Achyranthis bidentatae Radix (200 g) were purchased from Beijing Medicinal Herbs Co., Ltd. (Beijing, China), pulverized to a fine powder, suspended in distilled water to a concentration of $0.25 \mathrm{~g} / \mathrm{ml}$, and mixed well prior to administration. The representative chemical compositions of atractylenolide $(0.0085 \%)$, berberine $(0.734 \%)$, and $\beta$-ecdysone $(0.012 \%)$ in SM were determined by high-performance liquid chromatography analysis.

OA animal model and treatment. Ten-week-old male Sprague-Dawley rats (Animal Science Laboratory of Peking University Health Science Department, Beijing, China) were anesthetized with halothane, and subsequent to being shaved and disinfected, the right knee joint was exposed through a medial para-patellar approach. The patella was dislocated laterally and the knee placed in full flexion, followed by anterior cruciate ligament transection and medial meniscus resection (ACLT plus MMx) using micro-scissors. In the sham-operated negative controls, the right knee joint was exposed and incisions were closed following subluxation of the patella and washing of the joint surface with saline. The rats were randomly assigned to five groups: ACLT plus MMx without treatment (model, $n=12$ ), sham-operated (sham, $n=12$ ), and ACLT plus MMx rats treated with SM intragastrically at a daily dose of $0.63,1.25$ or $2.50 \mathrm{~g} / \mathrm{kg}$ (SM, n=12 per dose) for three or six weeks. Dose calculations followed guidelines correlating dose equivalents between humans and laboratory animals on the basis of ratios of body surface area (15); $2.5 \mathrm{~g} / \mathrm{kg}$ is the dose equivalent to a recommended dose of $0.4 \mathrm{~g} / \mathrm{kg}$ in humans. Rats in the model and sham groups received distilled water only. All animals were maintained on a $12 \mathrm{~h}$ light/dark cycle under constant temperature $\left(24 \pm 2^{\circ} \mathrm{C}\right)$ and humidity $(55 \pm 5 \%)$, and were allowed free access to food and water. All procedures for the consideration of animal welfare were reviewed and approved by the Ethical Committee of the China Academy of Chinese Medical Sciences (Beijing, China).

Histological analysis. Animals were sacrificed after three or six weeks of treatment. The tibia and femur were dissected and fixed in $4 \%$ paraformaldehyde for $24 \mathrm{~h}$, decalcified in $10 \%$ ethylenediamine tetraacetic acid (EDTA) and embedded in paraffin. Tissue sections $(4 \mu \mathrm{m})$ were mounted on common slides for staining with hematoxylin and eosin, toluidine blue and Masson's trichrome as described previously $(16,17)$. Cartilage histopathological features were analyzed using the scoring system modified by Mankin et al (18) (score range $0-12$, from normal to complete disorganization and hypocellularity). Synovium histopathology was evaluated according to Yoshimi's histological grading (score range 0-18, between normal and most severe reaction) (19). The Image-Pro Plus 6.0 System (IPP) image analysis system (Media Cybernetics, Rockville, MD, USA) was used for quantitative analysis. The positive index was calculated as the integral of the optical density. All sections were randomized and evaluated by a trained observer who was blinded to the treatment groups.

Terminal deoxynucleotidyl transferase-mediated dUTP nick end labeling (TUNEL). Apoptotic cells in specimens were recognized using In Situ Cell Apoptosis Detection kits (Boster Biological Technology, Ltd., Wuhan, China) according to the manufacturer's instructions (20). Briefly, deparaffinized sections were permeabilized in $0.1 \%$ Triton X-100, incubated in fluorescein-labeled dUTP and terminal deoxynucleotidyl transferase (TdT) mixture, then probed using an antifluorescein antibody conjugated with alkaline phosphatase. Sections were developed using substrate solution containing fast red. Sections without primary TdT were used as negative controls for the TUNEL staining. TUNEL-positive cells (apoptosis index, AI) in three different areas were counted under an Olympus OX31 microscope (Olympus, Tokyo, Japan). The Image-Pro Plus 6.0 System (IPP) image analysis system was used for quantitative analysis.

Immunoblotting analysis. Paraffin sections (4 $\mu \mathrm{m})$ of joint tissue were mounted on poly-L-lysine-coated slides. The paraffin sections were dewaxed by a routine method and incubated for 10 min with $3 \% \mathrm{H}_{2} \mathrm{O}_{2}$. Each section was incubated with blocking serum (Vectastain ${ }^{\circledR}$ ABC kit, Vector Laboratories, Burlingame, CA, USA) at room temperature for $30 \mathrm{~min}$ and then with primary rabbit monoclonal antibody against type II collagen (dilution 1/30; Beijing Biosynthesis Biotechnology Co., Ltd., Beijing, China), rabbit polyclonal antibody against MMP-13 (dilution 1/50; Abcam, Cambridge, UK) and rabbit monoclonal antibody against TIMP-1 (dilution 1/80; Abcam) overnight at $4^{\circ} \mathrm{C}$. Sections incubated in phosphate-buffered saline (PBS) without antibody served as negative controls. Following incubation with biotinylated secondary antibody (Zhonshan Golden Bridge Biotechnology, Beijing, China) and avidin-biotin complex reagent containing horseradish peroxidase (Vector Laboratories Ltd.) for $30 \mathrm{~min}$, the sections were then stained with 3,3'-diaminobenzidine (DAB; Sigma, St. Louis, MO, USA). The Image-Pro Plus 6.0 System (IPP) image analysis system was used for quantitative analysis.

Serum radioimmunoassay (RIA) and enzyme-linked immunosorbent assay (ELISA) analysis. Animal blood was collected from the abdominal aorta and the serum was analyzed for IL-1 $\beta$ and TNF- $\alpha$ by RIA, and for bone morphogenetic protein (BMP) and transforming growth factor- $\beta$ (TGF- $\beta$ ) by ELISA. NO and inducible NO synthase (iNOS) were detected by assay kits (Beijing 4A Biotech Co., Ltd., Beijing, China).

Chondrocyte culture and sample collection. Normal human chondrocytes were obtained commercially (ScienCell Research Laboratories, Carlsbad, CA, USA). The chondrocytes were maintained in a specific chondrocyte medium (ScienCell Research Laboratories) with 5\% heat-inactivated fetal bovine serum at $37^{\circ} \mathrm{C}$ in a humidified atmosphere of $95 \%$ air and $5 \%$ $\mathrm{CO}_{2}$. At the third passage, cells were seeded at a density of $1 \times 10^{5}$ cells per well in a six-well plate and cultured to $\sim 80 \%$ confluence, then made quiescent in serum-free medium for 
$24 \mathrm{~h}$. Chondrocytes were treated with SM at 0.83, 2.08 and $5.21 \mu \mathrm{g} / \mathrm{ml}$ for $1 \mathrm{~h}$, and then treated with IL-1 $\beta$ at $10 \mathrm{ng} / \mathrm{ml}$ for another $24 \mathrm{~h}$ of incubation. The controls consisted of chondrocytes incubated in the culture medium without IL-1 $\beta$ or SM. The chondrocytes were collected for further study.

Chondrocyte apoptosis by flow cytometry. To quantify the percentage of cells undergoing apoptosis, the Annexin V-FITC Apoptosis Detection kit (Nanjing KeyGen Biotech. Co., Ltd., Nanjing, China) was used according to the manufacturer's instructions. Briefly, chondrocytes were collected and the cells were washed twice with cold PBS. The cells were then resuspended in binding buffer at a concentration of $5 \times 10^{6}$ cells $/ \mathrm{ml}$. Following incubation, $100 \mathrm{ml}$ of the solution was transferred to a $5 \mathrm{ml}$ culture tube, and $5 \mu \mathrm{l}$ Annexin V-FITC and $10 \mu \mathrm{l}$ propidium iodide (PI) were added. The tube was gently vortexed and incubated for $15 \mathrm{~min}$ at room temperature in the dark. At the end of the incubation, $400 \mathrm{ml}$ binding buffer was added, and the cells were analyzed immediately by flow cytometry. A total of $1 \times 10^{6}$ chondrocytes were collected and the cells were fixed using cold $75 \%$ ethanol at $4{ }^{\circ} \mathrm{C}$ overnight, washed in PBS and incubated with $50 \mu \mathrm{g} / \mathrm{ml}$ PI and $5 \mu \mathrm{g} / \mathrm{ml}$ RNase at room temperature for $30 \mathrm{~min}$. The cell cycle analysis was performed using flow cytometry (provided by the Department of Cell Biology, School of Medicine, Shanghai Jiao Tong University, Shanghai, China).

Western blot analysis. Following treatment, all chondrocytes were harvested in cold PBS. The pellet was resuspended in lysis buffer (50 mm Tris $\mathrm{pH} 8.0,150 \mathrm{~mm} \mathrm{NaCl}, 5 \mathrm{~mm}$ EDTA, $0.1 \%$ sodium dodecyl sulfate, $0.5 \%$ nonyl phenoxypolyethoxylethanol-40) containing $10 \mathrm{~mm}$ phenylmethylsulfonyl fluoride and $2 \mathrm{mg} / \mathrm{ml}$ aprotinin. Protein was obtained to detect the levels of MMP-13 and TIMP-1 in the chondrocytes by western blotting. The western blotting protocol and semi-quantitative analysis were carried out as described previously (21). The following antibodies were used: Rabbit anti-MMP-13 monoclonal antibody (dilution 1/100, Abcam), rabbit anti-TIMP-1 polyclonal antibody (dilution 1/50, Boster Biological Technology, Ltd.) and GAPDH antibody (internal control, rabbit polyclonal antibody, dilution 1/200, Santa Cruz Biotechnology, Inc., Santa Cruz, CA, USA). All experiments were carried out in triplicate. The relative quantity of each antibody was measured using AlphaEase FC (FluorChem FC2) software (Cell Biosciences Inc., Santa Clara, CA, USA). The density ratio of protein to GAPDH was calculated from the band density.

Quantitative polymerase chain reaction ( $q P C R$ ). Following treatment, total RNA was extracted from the chondrocytes with TRIzol ${ }^{\circledR}$ reagent (Invitrogen Life Sciences, Carlsbad, CA, USA) according to the manufacturer's instructions. The total RNA $(2 \mu \mathrm{g})$ was reverse transcribed to cDNA using the High Capacity cDNA Reverse Transcription kit (Applied Biosystems, Inc., Foster City, CA, USA) according to the manufacturer's instructions. The specific transcripts were quantified by qPCR using a QuantiTect SYBR Green PCR kit (Qiagen, Tokyo, Japan) and analyzed with an Applied Biosystems ${ }^{\circledR} 7500$ Fast Real-Time PCR system (Applied Biosystems Inc.). Gene-specific primers were used for MMP-13 (forward,
TGAGGATACAGGCAAGACTCT; reverse, CAATACGGT TACTCCAGATGC), TIMP-1 (forward, CTTCTGGCATCC TGTTGTTG; reverse, AGAAGGCCGTCTGTGGGT) and GAPDH (forward, GAAGGTGAAGGTCGGAGTC; reverse, GAAGATGGTGATGGGATTTC). The mRNA levels of MMP-13 and TIMP-1 were normalized to the GAPDH mRNA level. PCR was performed at $95^{\circ} \mathrm{C}$ for $10 \mathrm{~min}$, followed by 40 cycles of $95^{\circ} \mathrm{C}$ for $30 \mathrm{sec}$ and $60^{\circ} \mathrm{C}$ for $1 \mathrm{~min}$. The quantification data was analyzed with ABI Prism ${ }^{\circledR}$ analysis software (Applied Biosystems, Inc.). The relative mRNA expression was calculated with the comparative threshold cycle method (22).

Statistical analysis. All data are expressed as the mean \pm standard deviation. The contents of collagen and proteoglycans were analyzed with non-parametric statistics (Kruskal-Wallis one-way analysis of variance). The other data were analyzed using a two-tailed Student's t-test, two-sample assuming unequal variance, within the MS Excel 2007 software package (Microsoft Corp., Redmond, WA, USA). Values were considered as significantly different if $\mathrm{P}<0.05$.

\section{Results}

Effects of SM on the histopathology of articular cartilage and synovium in the knee joints of ACLT plus Mmx rats. To evaluate the anti-OA effect of SM, the isolated knee joints from the five groups were analyzed microscopically. The articular cartilage histopathological results are shown in Fig. 1. The sham group rats showed smoothly surfaced articular cartilage and normal cellularity. By contrast, the knee joints from the untreated rats with ACLT plus MMx-induced OA revealed significant ongoing histopathological changes, as indicated by surface irregularity, disorganization of the articular cartilage with apparent cloning of chondrocytes in the transitional and radial zones, and a disrupted tidemark. As expected, the overall Mankin score was significantly increased by ACLT plus MMx. These histomorphological changes in the cartilage were reduced in the SM-treated OA animals, particularly with SM at the dose of $2.5 \mathrm{~g} / \mathrm{kg}$, which significantly restored cartilage morphology as indicated by normal cartilage surfaces with normal cellularity in the transitional and radial zones, and a normal tidemark. SM $(2.5 \mathrm{~g} / \mathrm{kg})$ significantly decreased the overall modified Mankin scores compared with those of the untreated OA model rats at three and six weeks of treatment (both $\mathrm{P}<0.001$ ).

Histopathological features of the synovium are shown in Fig. 2. The untreated ACLT plus Mmx model rats exhibited increased hyperplasia of synovial lining cells, hypertrophy of the synovial lining layer and an increase in the infiltration of inflammatory cells in synovial tissue compared with that observed in the sham rats. SM $(2.5 \mathrm{~g} / \mathrm{kg})$ suppressed hypertrophy of the synovial lining layer and inflammatory cellular infiltration, and significantly reduced the histological severity scores in rats following ACLT plus Mmx compared with those in the untreated ACLT plus Mmx model rats at three and six weeks of treatment $(\mathrm{P}<0.05$ and $\mathrm{P}<0.01$, respectively).

The above results provide evidence that SM exhibits significant anti-OA activity. Notably, the largest changes in these parameters were identified at the highest dose. These data prompted further studies to elucidate the mechanism of SM $(2.5 \mathrm{~g} / \mathrm{kg})$ activity. 

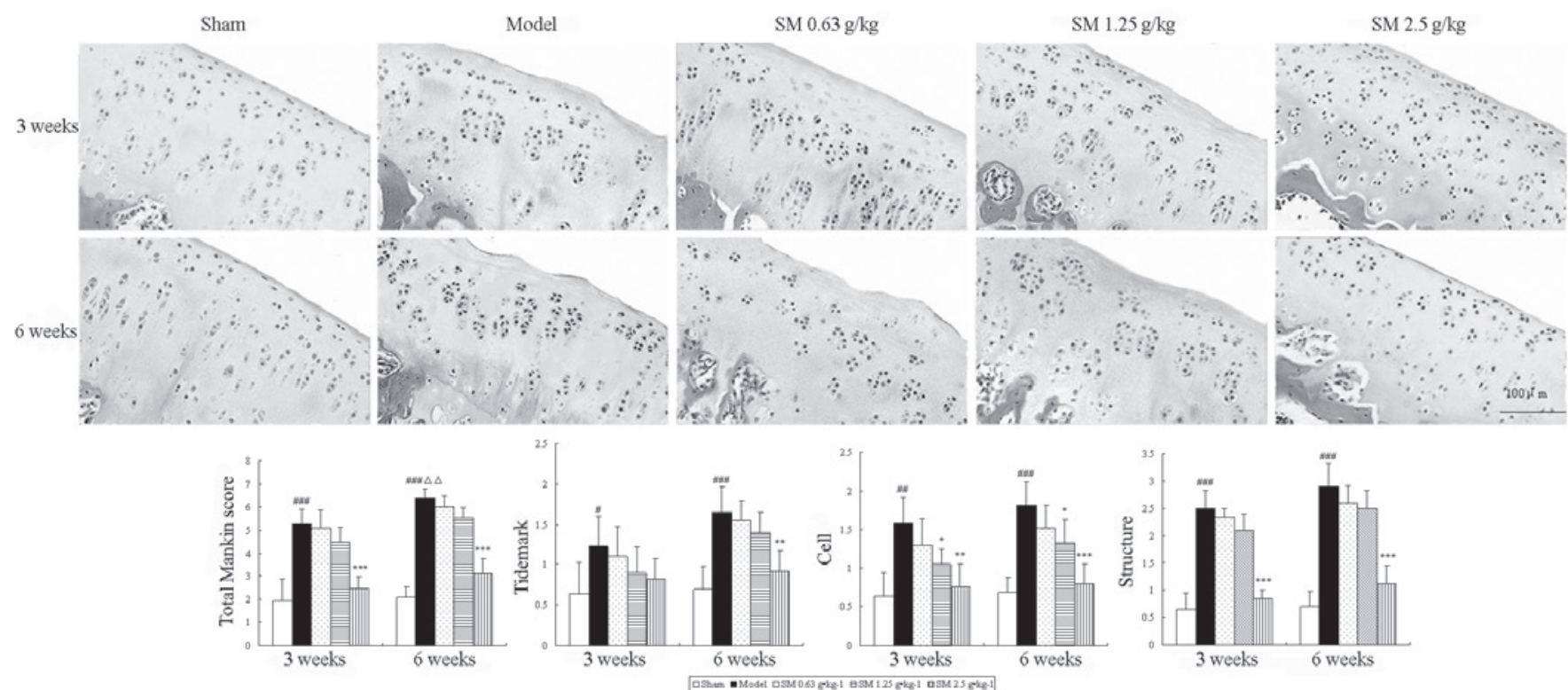

Figure 1. Effects of SM on cartilage histology and Mankin score in the knee joints of rats following ACLT plus Mmx. Cartilage histology was observed in ACLT plus MMx model and sham-operated rats at the end of a three- or six-week treatment period. Photomicrographs captured at $x 200$ magnification of representative cartilage sections from one animal per treatment group are shown: Sham, untreated ACLT plus MMx (model) and ACLT plus MMx treated with $\mathrm{SM}$ at the doses of $0.63,1.25$ and $2.5 \mathrm{~g} / \mathrm{kg}(\mathrm{SM})$. At three or six weeks, significant histopathological changes were evident in the model rats, as indicated by surface irregularity, disorganization of articular cartilage with apparent cloning of chondrocytes in the transitional and radial zones, and an intact tidemark. Treatment with SM $(2.5 \mathrm{~g} / \mathrm{kg})$ significantly prevented damage to the cartilage structure, reduced cellular abnormalities, and prevented change of the tidemark. The Mankin scores of the SM $(2.5 \mathrm{~g} / \mathrm{kg})$ group were significantly decreased compared with those of the model group. Data are presented as the mean \pm standard deviation of samples from six rats per group. P-values are from the Student's t-test comparing the SM group with the model group. ${ }^{*} \mathrm{P}<0.05,{ }^{* * *} \mathrm{P}<0.01$ and ${ }^{* * * *} \mathrm{P}<0.001$ compared with the model group; ${ }^{\#} \mathrm{P}<0.05,{ }^{\# \#} \mathrm{P}<0.01$ and ${ }^{\# \# \prime} \mathrm{P}<0.001$ compared with the sham group; ${ }^{\Delta \Delta} \mathrm{P}<0.01$ compared with the model group at the three weeks. SM, SianMiao formula; ACLT plus MMx, anterior cruciate ligament transection and medial meniscus resection.
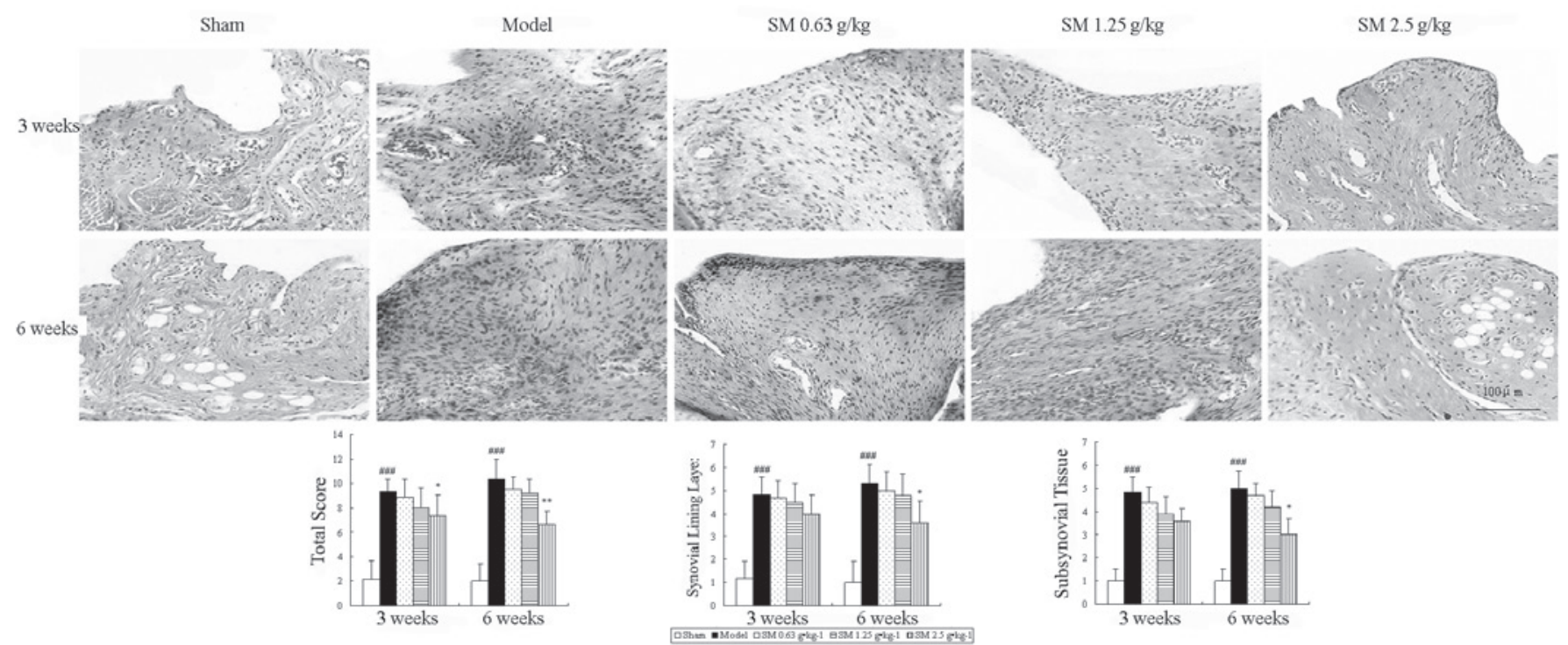

Figure 2. Effects of SM on synovial histology and total score in the knee joints of rats following ACLT plus Mmx. Synovial histology was observed in ACLT plus MMx model and sham-operated rats at the end of a three- or six-week treatment period. Photomicrographs were captured at x200 magnification of representative cartilage sections from one animal per group. Untreated ACLT plus Mmx model rats exhibited increased hypertrophy and infiltration of inflammatory cells in synovial tissue compared with sham rats. SM $(2.5 \mathrm{~g} / \mathrm{kg})$ significantly inhibited cellular infiltration and the histological severity scores in rats following ACLT plus Mmx. Data are presented as the mean \pm standard deviation of samples from six rats per group. P-values are from the one-way analysis of variance. ${ }^{*} \mathrm{P}<0.05$ and ${ }^{* *} \mathrm{P}<0.01$ compared with the model group; ${ }^{\# \#} \mathrm{P}<0.001$ compared with the sham group. SM, SianMiao formula; ACLT plus $\mathrm{MMx}$, anterior cruciate ligament transection and medial meniscus resection.

Effects of SM on chondrocyte apoptosis in the knee joints of ACLT plus Mmx rats. Apoptotic chondrocytes were observed using the TUNEL assay, and the results are shown in Fig. 3. Increased numbers of apoptotic chondrocytes were observed in the cartilage of untreated ACLT plus Mmx model rats and the percentages of apoptotic chondrocytes were 29.5 and $40.75 \%$ at three and six weeks, respectively. Compared with the untreated model group, SM $(2.5 \mathrm{~g} / \mathrm{kg})$ treatment signifi- 


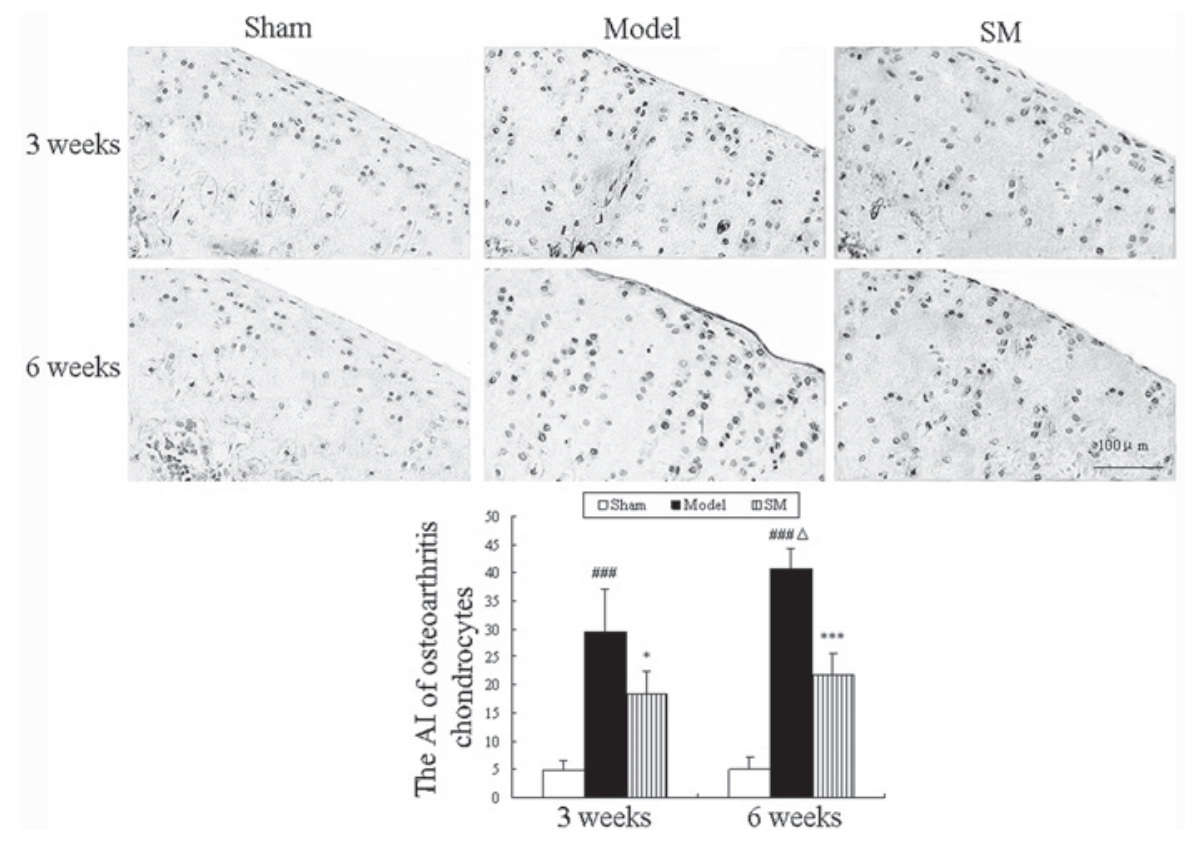

Figure 3. Effects of SM on chondrocyte apoptosis in the knee joints of rats following anterior cruciate ligament transection and medial meniscus resection. Apoptosis of chondrocytes was examined via DNA fragmentation using a terminal deoxynucleotidyl transferase-mediated dUTP nick end labeling assay. Photomicrographs were captured at x 200 magnification of representative cartilage sections from one animal per group. Data are presented as the mean \pm standard deviation of samples from six rats in each group. ${ }^{*} \mathrm{P}<0.05$ and ${ }^{* * *} \mathrm{P}<0.001$ compared with the model group; ${ }^{\# \# \#} \mathrm{P}<0.001$ compared with the sham group; ${ }^{\Delta} \mathrm{P}<0.05$ compared with the model group at three weeks. SM, SanMiao formula; AI, apoptosis index.

cantly decreased the percentage of apoptotic chondrocytes at three and six weeks $(\mathrm{P}<0.05$ and $\mathrm{P}<0.01$, respectively) and caused a $19 \%$ reduction in the proportion of TUNEL-positive cells among the total cells at six weeks compared with that in the untreated group.

Effects of SM on cartilage matrix in the knee joints of ACLT plus Mmx rats. Proteoglycan and collagen are major constituents of articular cartilage matrix, which were evaluated by toluidine blue and Masson's trichrome staining. The control group showed intensive toluidine blue and Masson's trichrome staining. In the untreated ACLT plus Mmx model rats, toluidine blue and Masson's trichrome staining shown a significant ongoing reduction, which indicated loss of proteoglycan and collagen. Furthermore, immunohistochemistry revealed a significant reduction in type II collagen levels. Treatment with SM $(2.5 \mathrm{~g} / \mathrm{kg})$ significantly inhibited cartilage matrix degradation, as indicated by attenuation of the loss of proteoglycan and collagen and upregulation of the expression of type II collagen at three and six weeks. SM $(2.5 \mathrm{~g} / \mathrm{kg})$ induced $\sim 2.3-, 1.9-$ and 2.5 -fold increases in the expression levels of proteoglycan $(\mathrm{P}<0.001)$, collagen $(\mathrm{P}<0.05)$ and type II collagen $(\mathrm{P}<0.001)$, respectively, compared with those of untreated ACLT plus Mmx model rats at six weeks (Fig. 4).

Effects of SM on MMP-13 and TIMP-1 expression levels in the knee joints of ACLT plus Mmx rats. The effects of SM $(2.5 \mathrm{~g} / \mathrm{kg})$ treatment on the expression levels of MMP-13 and TIMP-1 in rats following ACLT plus Mmx were assessed by immunohistochemistry. Representative joint sections from all groups and the findings from the quantitative analysis are shown in Fig. 5. ACLT plus MMx induced increased the expression levels of MMP-13 and decreased the expression levels of TIMP-1 in the joint. Treatment with SM $(2.5 \mathrm{~g} / \mathrm{kg})$ induced significant differences compared with untreated ACLT plus Mmx model rats at three and six weeks. SM decreased the expression of MMP-13 by $39 \%(\mathrm{P}<0.001)$ and increased that of TIMP-1 by $51 \%$ $(\mathrm{P}<0.01)$ compared with that in the untreated ACLT plus Mmx model rats at six weeks. MMP-13 and TIMP-1 were expressed in the chondrocytes of the knee joint in all groups. The positive staining was predominantly cytoplasmic or cytosolic in the chondrocytes. These results support the indication that SM mediates anti-OA activity through the MMP/TIMP system.

Effects of SM on the levels of $I L-1 \beta, T N F-a, N O, i N O S$, BMP and TGF- $\beta$ in the serum of ACLT plus Mmx rats. To determine whether SM induced changes in the levels of inflammation-related cytokines, chondrocytes and cartilage matrix metabolism-related cytokines in serum, the levels of IL- $1 \beta$, TNF- $\alpha$, NO, iNOS, BMP and TGF- $\beta$ in serum were examined. The serum levels of IL-1 $\beta$, TNF- $\alpha$, NO and iNOS exhibited a significant ongoing increase. This was significantly suppressed by SM with the following reductions at six weeks of treatment: IL- $1 \beta, 48 \%, \mathrm{P}<0.05$; TNF- $\alpha, 31 \%, \mathrm{P}<0.05$; NO, $18 \%$, $\mathrm{P}<0.01$ and iNOS, $23 \%, \mathrm{P}<0.05$. The levels of BMP and TGF- $\beta$ were significantly decreased in the model group compared with those in the sham group during the treatment period. Treatment with SM $(2.5 \mathrm{~g} / \mathrm{kg})$ caused significant increases in the levels of BMP-2 $(\mathrm{P}<0.05)$ and TGF- $\beta_{1}(\mathrm{P}<0.01)$, with 55 and $43 \%$ increases respectively at six weeks of treatment (Fig. 6).

Effects of SM on chondrocyte apoptosis in the flow cytometric analysis. IL-1 $\beta$ increased the proportion of apoptotic sub-G1 phase cells. SM significantly decreased the proportion of apoptotic sub-G1 phase cells in a concentration-dependent manner as indicated by PI staining using flow cytometry. SM at $5.21 \mu \mathrm{g} / \mathrm{ml}$ 

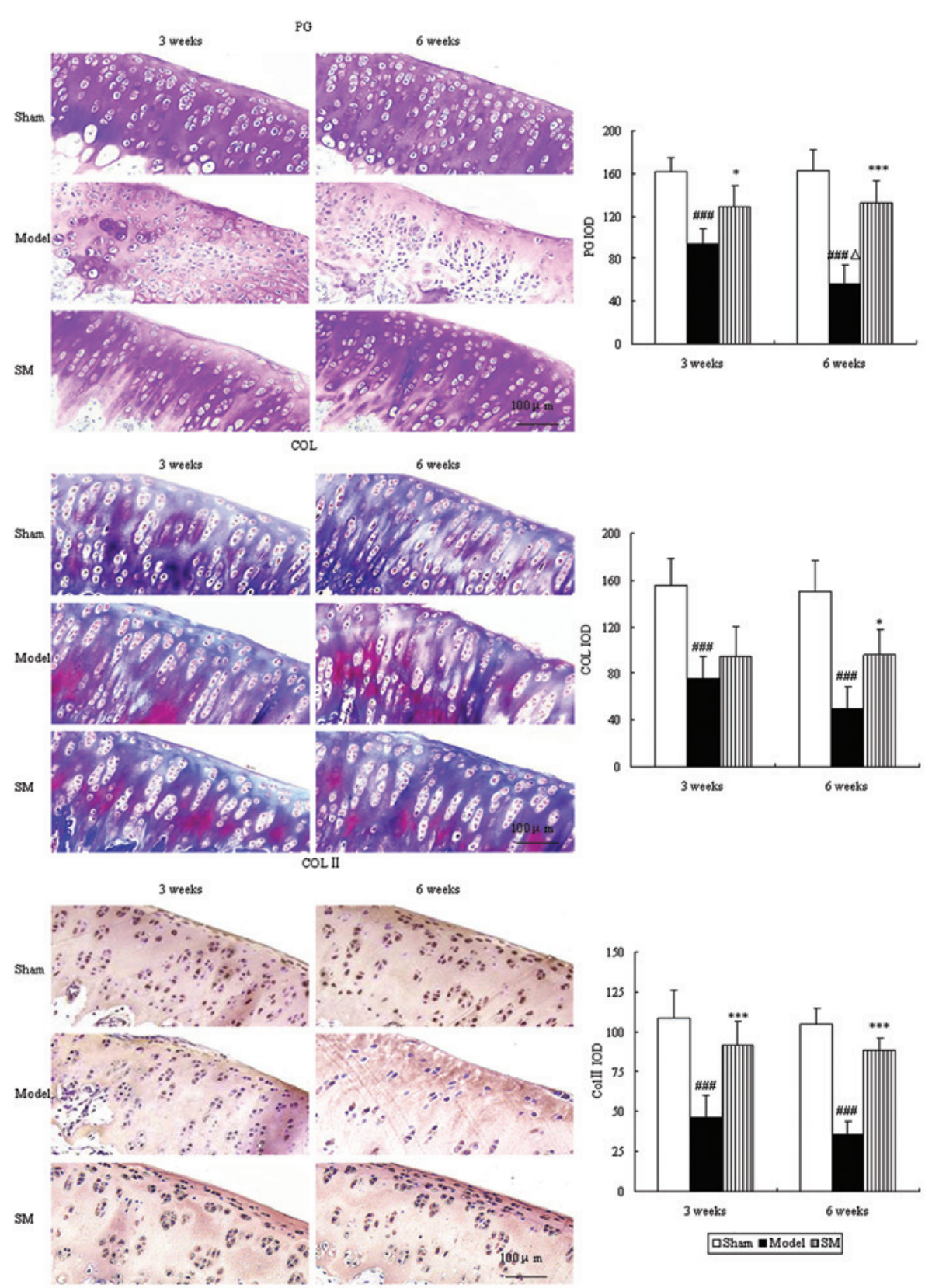

Figure 4. Effects of SM on cartilage matrix components in the knee joints of rats following anterior cruciate ligament transection and medial meniscus resection. The effects of SM on PG, COL and Col II. Toluidine blue, Masson's staining and immunohistochemistry were used to assess PG, COL and COL II. Photomicrographs were captured at x200 magnification of representative cartilage sections from one animal per group. Data are presented as the mean \pm standard deviation of samples from six rats in each group. ${ }^{*} \mathrm{P}<0.05$ and ${ }^{* * *} \mathrm{P}<0.001$ compared with the model group; ${ }^{\# \# \# ~} \mathrm{P}<0.001$ compared with the sham group; ${ }^{\wedge} \mathrm{P}<0.05$ compared with the model group at three weeks. SM, SanMiao formula; PG, proteoglycan; COL, collagen; COL II, type II collagen; IOD, integrated optical density.

induced a $32.38 \%$ reduction in apoptotic sub-G1 phase cells (Fig. 7A). The inhibitory effect of SM on apoptosis was confirmed using Annexin V staining to detect the externalization of phosphatidylserine on the cell membrane. SM markedly decreased early and late apoptotic cells, and SM at $5.21 \mu \mathrm{g} / \mathrm{ml}$ induced a $34 \%$ reduction in early and late apoptotic cells (Fig. 7B).

Effects of SM on the production and gene expression of MMP-13 and TIMP-1 in human chondrocytes. The expression of MMP-13 was significantly downregulated when the chondrocytes were incubated with SM at $0.83,2.08$ or $5.21 \mu \mathrm{g} / \mathrm{ml}$. The downregulating effects were augmented as the concentration of
SM increased, and SM at $5.21 \mu \mathrm{g} / \mathrm{ml}$ induced a $49 \%$ reduction in the level of MMP-13 $(\mathrm{P}<0.001)$ compared with that in untreated IL-1 $\beta$-induced chondrocytes. The expression of TIMP-1 was clearly upregulated following incubation with SM at all three concentrations. The largest increases were found at $5.21 \mu \mathrm{g} / \mathrm{ml}$ $\mathrm{SM}(\mathrm{P}<0.001)$ with a 1.6 -fold upregulation (Fig. 8A). To further validate the above mechanisms, the effects of SM on the mRNA expression of MMP-13 and TIMP-1 in IL-1 $\beta$-induced chondrocytes were also assessed by qPCR. SM significantly inhibited the IL-1 $\beta$-induced gene expression of MMP-13 in a concentration-dependent manner. SM at the highest concentration induced a 57\% reduction in MMP-13 mRNA levels $(\mathrm{P}<0.01)$ 

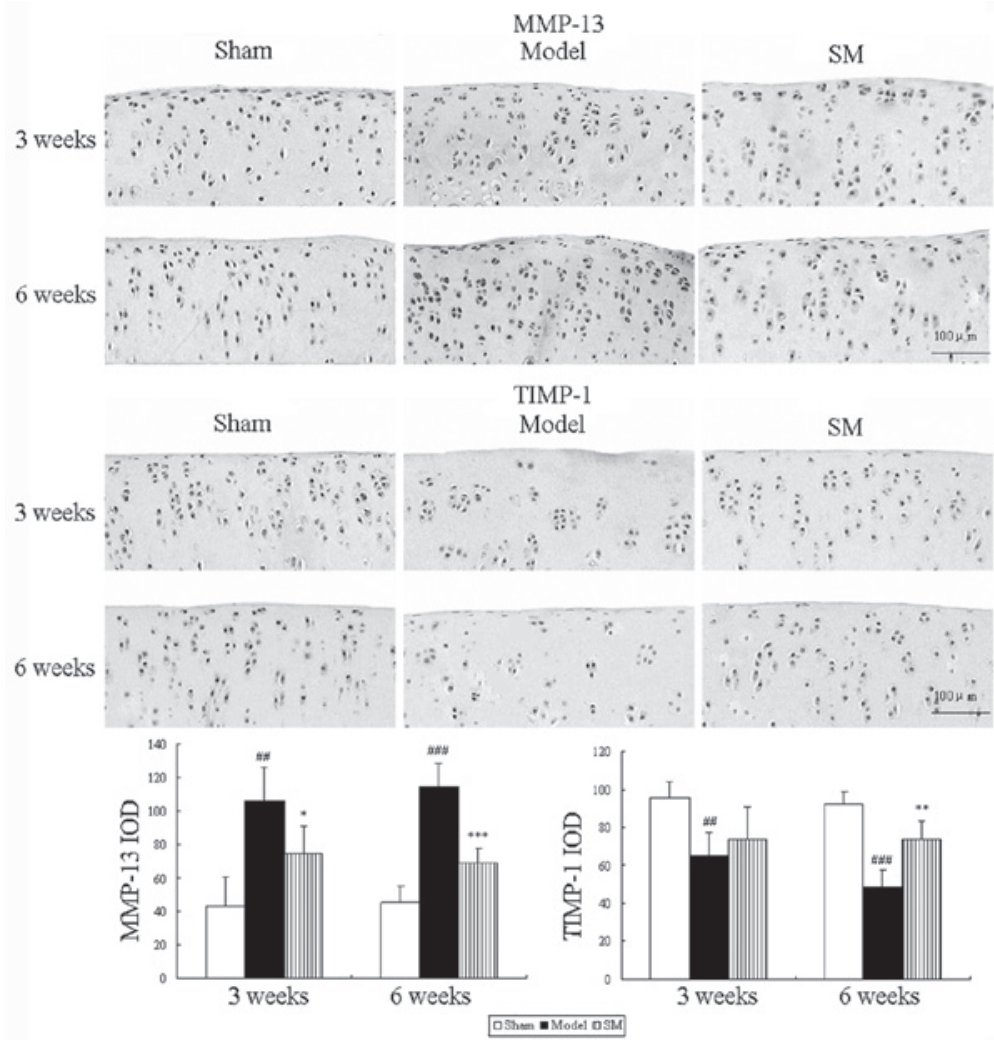

Figure 5. Effects of SM on the expression levels of MMP-13 and TIMP-1 in the knee joints of rats with anterior cruciate ligament transection and medial meniscus resection. The expression levels of these enzymes were assessed by quantitative immunohistochemistry, Photomicrographs were captured at $\mathrm{x} 200$ magnification of representative cartilage sections from one animal per group. Quantitative immunohistochemistry analyses of the expression levels of these enzymes in the tibia are shown. Data are presented as the mean \pm standard deviation of samples from six rats in each group. ${ }^{*} \mathrm{P}<0.05,{ }^{* * *} \mathrm{P}<0.01$ and ${ }^{* * *} \mathrm{P}<0.001$ compared with the model group; ${ }^{\# \#} \mathrm{P}<0.01$ and ${ }^{\# \# "} \mathrm{P}<0.001$ compared with the sham group; SM, SanMiao formula; MMP-13, matrix metalloproteinase-13; TIMP-1, tissue inhibitor of metalloproteinase-1; IOD, integrated optical density.
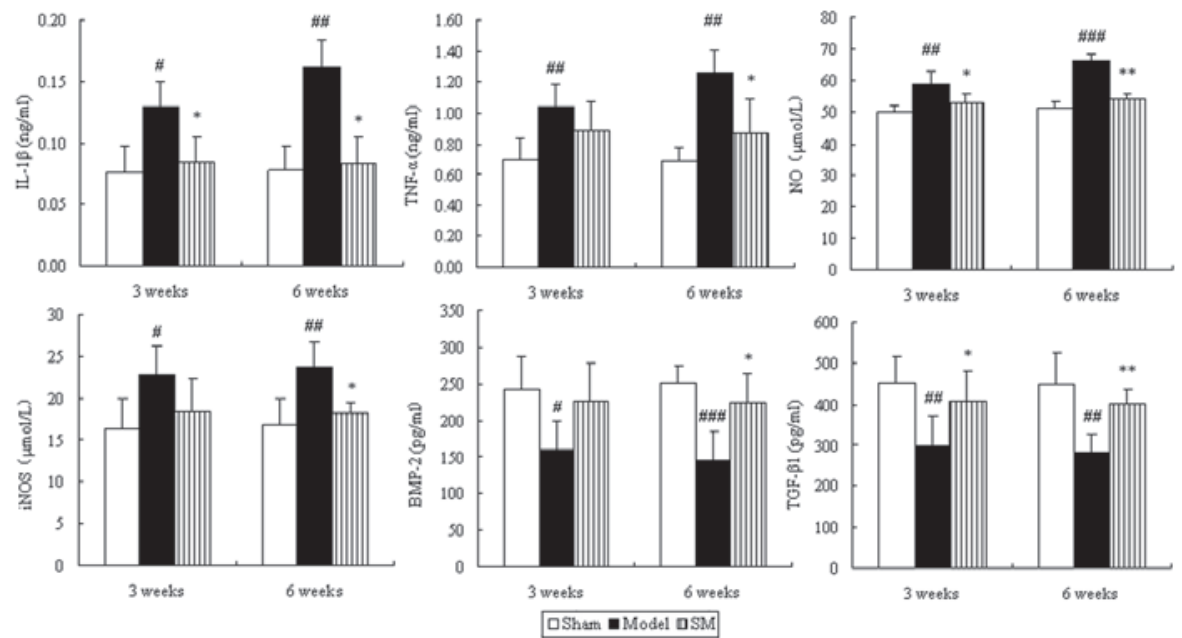

Figure 6. Effects of SM on IL-1 $\beta$, TNF- $\alpha$, NO, iNOS, BMP-2 and TGF- $\beta_{1}$ in the serum of rats with osteoarthritis. Blood was obtained from the abdominal aorta at three and six weeks of treatment following the anterior cruciate ligament transection and medial meniscus resection and sham surgeries. The serum was used for the assays of IL- $1 \beta$, TNF- $\alpha$, NO, iNOS, BMP-2 and TGF- $\beta_{1}$. Data are presented as the mean \pm standard deviation of samples from six rats in each group. ${ }^{*} \mathrm{P}<0.05$ and ${ }^{* *} \mathrm{P}<0.01$ compared with the model group; ${ }^{\#} \mathrm{P}<0.05,{ }^{\# \#} \mathrm{P}<0.01$ and ${ }^{\# \# \#} \mathrm{P}<0.001$ compared with the sham group. SM, SanMiao formula; IL-1 $\beta$, interleukin-1 $\beta$; TNF- $\alpha$, tumor necrosis factor- $\alpha$; NO, nitric oxide; iNOS, inducible NO synthase; BMP-2, bone morphogenetic protein-2; TGF- $\beta_{1}$; transforming growth factor- $\beta_{1}$.

compared with those in the model group. SM upregulated the gene expression of TIMP-1, and SM at $5.21 \mu \mathrm{g} / \mathrm{ml}$ induced a 1.3-fold upregulation in TIMP-1 mRNA levels compared with those in the model group $(\mathrm{P}<0.001 ;$ Fig. 8B).

\section{Discussion}

$\mathrm{OA}$ is a degenerative joint disease with multiple underlying pathogenic mechanisms caused by various risk factors. The 
A

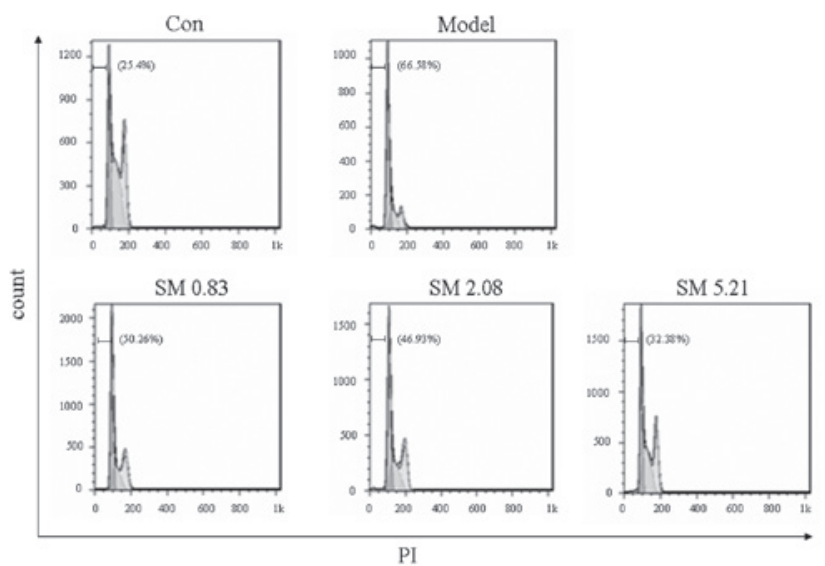

B

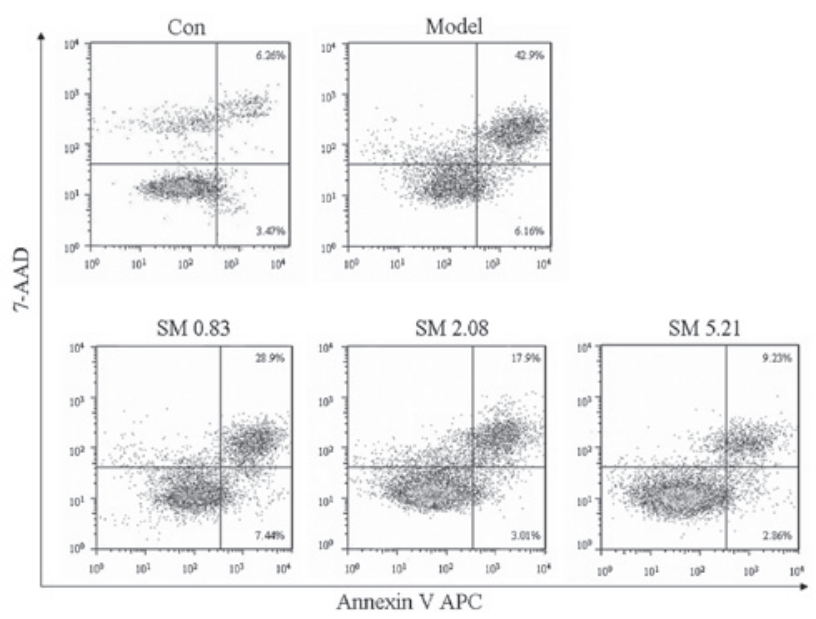

Figure 7. Effects of SM on apoptosis in human chondrocytes. (A) Effects of SM on apoptotic G1 phase cells in chondrocytes treated with $10 \mathrm{ng} / \mathrm{ml}$ interleukin-1 $\beta$ were analyzed by flow cytometric analysis. (B) Effects of SM on apoptosis were analyzed by Annexin V/PI staining using flow cytometric analysis. Chondrocytes were treated with SM at $0.83,2.08$ and $5.21 \mu \mathrm{g} / \mathrm{ml}$. The percentage data were obtained from three independent experiments. SM, SanMiao formula; PI, propidium iodide; 7-AAD, 7-aminoactinomycin D.

A

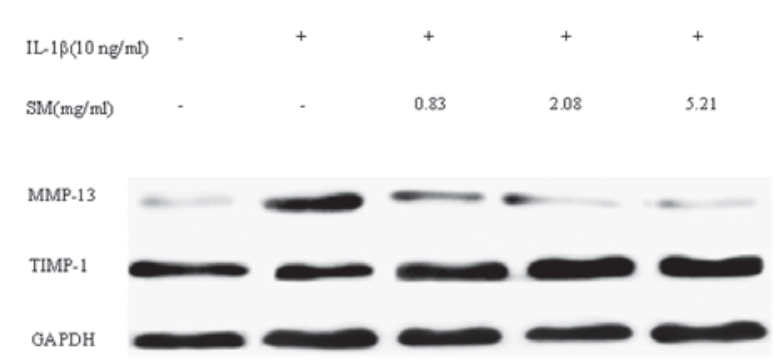

DSham $\boldsymbol{M}$ Model OSM 0.83 OSM 2.08 ESM S.21

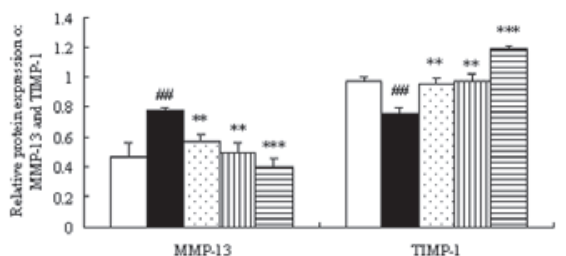

B
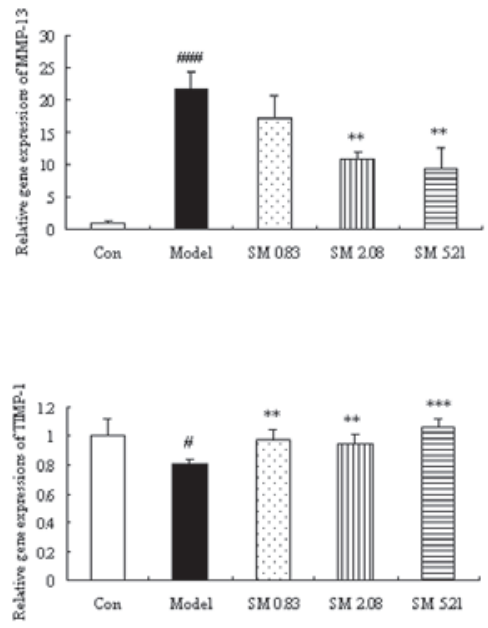

Figure 8. Effects of SM on the production and gene expression of MMP-13 and TIMP-1 in IL-1 $\beta$-induced human chondrocytes. (A) The relative protein expressions of MMP-13 and TIMP-1 in IL-1 $\beta$-induced human chondrocytes by western blot analysis. Representative blots are shown in the upper panel, and the quantitative analyses are shown below. (B) Relative gene expression of MMP-13 and TIMP-1 in IL-1 $\beta$-induced human chondrocytes as evaluated by quantitative polymerase chain reaction analysis. Data are presented as the mean \pm standard deviation. ${ }^{* *} \mathrm{P}<0.01$ and ${ }^{* * *} \mathrm{P}<0.001$ compared with the model group; ${ }^{\#} \mathrm{P}<0.05,{ }^{\# \#} \mathrm{P}<0.01$ and ${ }^{\# \# /} \mathrm{P}<0.001$ compared with the control group. Con, control; SM, SanMiao formula; MMP-13, matrix metalloproteinase-13; TIMP-1, tissue inhibitor of metalloproteinase-1; IL-1 $\beta$, interleukin-1 $\beta$. 
loss of chondrocyte function, degradation of the ECM and inflammation play crucial roles in OA progression, and thus inhibiting the development of these factors is an important issue for the treatment of OA. To the best of our knowledge, the effects of SM on OA have not been characterized. In the present study, the anti-OA effect of SM was evaluated using a classical ACLT plus MMx rat model. The new findings demonstrate than SM significantly inhibited articular cartilage damage and synovial inflammation in rats following ACLT plus Mmx, by inhibiting chondrocyte apoptosis, cartilage matrix degradation and the release of proinflammatory cytokines and inflammatory mediators.

Chondrocyte apoptosis plays an key role in the degeneration and degradation of articular cartilage in cases of OA. Reduced cellularity is a characteristic feature of OA cartilage and apoptosis has been proposed as an underlying cause of the hypocellularity (23). In the present study, it was shown that the cell scores in knee cartilage following ACLT plus MMx were markedly increased compared with those in nonarthritic control cartilage, and that cell scores correlated positively with the percentage of apoptotic chondrocytes, suggesting that the reduced cellularity in OA cartilage may be at least partially attributable to cell death by apoptosis. The results from the TUNEL analysis showed that SM suppressed chondrocyte apoptosis in the superficial and middle zones of cartilage of ACLT plus Mmx rats. These results are confirmed by the in vitro experiments in the present study, which revealed that SM inhibited the increase in the proportion of early and late apoptotic cells and sub-G1 phase cells in IL-1 $\beta$-induced chondrocytes, suggesting that SM prevents the degeneration and degradation of articular cartilage by inhibiting chondrocyte apoptosis.

Cartilage matrix consists largely of proteoglycan and collagen; the loss of proteoglycan occurs early in the process of cartilage degeneration and is followed by the catabolism of collagen fibrils (24). The tensile strength of articular cartilage is provided by a network of fibrils which exhibit a slow turnover that may occur over years, rendering collagen damage a particular problem (25). Type II collagen is known to be the most common type of collagen in hyaline articular cartilage (26). MMP-13, a primary collagenase in OA, degrades type II collagen and is inhibited by TIMP-1 $(27,28)$. Under normal conditions, TIMPs bind to active MMPs in a 1:1 ratio to make an inactive complex. An imbalance in the ratio of TIMPs to MMPs causes the continued matrix destruction in OA (11). In the present study, it was demonstrated that SM significantly interfered with the OA-induced expression of MMP-13, while augmenting that of TIMP-1 in the joints of OA model rats. These results are supported by the in vitro experiments in the present study, which revealed that the IL-1 $\beta$-augmented expression of MMP-13 was inhibited and the protein and gene expression levels of TIMP-1 were upregulated by SM in the IL-1 $\beta$-induced chondrocytes. A previous study indicated that the important ingredient berberine in SM decreased glycosaminoglycan release, inhibited the expression of MMP-1, -3 and -13 , and increased the level of TIMP-1 at the mRNA level in IL-1 $\beta$-induced rat articular chondrocytes (29). Therefore, regulating the balance of MMPs/TIMPs is one of the mechanisms by which SM prevents cartilage matrix degradation.

The progression of $\mathrm{OA}$ is currently considered to be associated with inflammation in the early stages of the disease. Among the proinflammatory cytokines involved in OA, IL-1 $\beta$ and TNF- $\alpha$ are considered the major participants. In patients or animals with OA, levels of IL-1 $\beta$ and TNF- $\alpha$ are elevated in the synovial fluid and serum (30). NO, a gaseous free radical, is an important inducer of apoptosis as well as the upregulation of MMP-13 (31) and is synthesized by iNOS. The results of the present study showed that SM significantly decreased the secretion of IL-1 $\beta$ and TNF- $\alpha$ in serum. Additionally, SM markedly inhibited the release of $\mathrm{NO}$ in the circulation through suppression of iNOS production. Sun et al (32) reported that SM effectively inhibited arthrocele and synovial inflammation in rats with adjuvant arthritis (AA). Thus, SM may be able to suppress the inflammatory response of arthritis. However, the dose of SM used in the rats with AA was higher than that used in the OA model rats in the present study. All of the three ingredients of SM contain anti-inflammatory substances, including berberine and $\beta$-eudesmol $(33,34)$. SM may inhibit the inflammatory response in OA by interfering with the secretion of proinflammatory cytokines and inflammatory mediators. TGF- $\beta$ is one of the most potent mediators of cartilage matrix synthesis (35). It upregulates the expression of several types of collagens and proteoglycans. Other members of the TGF- $\beta$ superfamily, BMPs, are also known to stimulate cartilage matrix synthesis. In particular, BMP-2 has been shown to stimulate the anabolic activity of chondrocytes as well as being present in articular cartilage (36). The results of the present study revealed that SM may exert anti-OA activity by upregulating the levels of TGF- $\beta$ and BMP-2.

In conclusion, $\mathrm{SM}$ effectively exerted anti-OA activity in ACLT plus Mmx model rats. The effect of prevention of cartilage articular damage was mainly via the direct suppression of chondrocyte apoptosis and cartilage matrix degradation, and interference with the secretion of proinflammatory cytokines and inflammatory mediators as well as chondrocytes and cartilage matrix metabolism-related cytokines in the OA model rats. Furthermore, the blocking of chondrocyte apoptosis was achieved by inhibiting the proportion of early and late apoptotic and sub-G1 phase cells. The effect of preventing cartilage matrix degradation was achieved by the suppression of MMP-13 expression, simultaneous upregulation of TIMP-1 production, and maintenance of the MMP to TIMP balance in the knee joints and chondrocytes. This study, to the best of our knowledge, provides the first evidence that SM can effectively treat OA, as part of an ongoing effort to identify novel and potent agents for the prevention and treatment of OA.

\section{Acknowledgements}

This study was supported by grants from the project of the National Natural Science Foundation of China (no. 81072900).

\section{References}

1. Loeser RF: Aging and osteoarthritis: the role of chondrocyte senescence and aging changes in the cartilage matrix. Osteoarthritis Cartilage 17: 971-979, 2009.

2. Goldring MB: The role of the chondrocyte in osteoarthritis. Arthritis Rheum 43: 1916-1926, 2000.

3. Roughley PJ: Articular cartilage and changes in arthritis: noncollagenous proteins and proteoglycans in the extracellular matrix of cartilage. Arthritis Res 3: 342-347, 2001 
4. Aigner T and Kim HA: Apoptosis and cellular vitality: issues in osteoarthritic cartilage degeneration. Arthritis Rheum 46: 1986-1996, 2002.

5. Eyre D: Collagen of articular cartilage. Arthritis Res 4: 30-35, 2002.

6. Kapoor M, Martel-Pelletier J, Lajeunesse D, Pelletier JP and Fahmi H: Role of proinflammatory cytokines in the pathophysiology of osteoarthritis. Nat Rev Rheumatol 7: 33-42, 2011.

7. Aizawa T, Kon T, Einhorn TA and Gerstenfeld LC: Induction of apoptosis in chondrocytes by tumor necrosis factor-alpha. J Orthop Res 19: 785-796, 2001.

8. Fernandes JC, Martel-Pelletier J and Pelletier JP: The role of cytokines in osteoarthritis pathophysiology. Biorheology 39: 237-246, 2002.

9. Attur M, Al-Mussawir HE, Patel J, et al: Prostaglandin E2 exerts catabolic effects in osteoarthritis cartilage: evidence for signaling via the EP4 receptor. J Immunol 181: 5082-5088, 2008.

10. Abramson SB: Osteoarthritis and nitric oxide. Osteoarthritis Cartilage 16 (Suppl 2): S15-S20, 2008.

11. Burger D, Rezzonico R, Li JM, et al: Imbalance between interstitial collagenase and tissue inhibitor of metalloproteinases 1 in synoviocytes and fibroblasts upon direct contact with stimulated T lymphocytes: involvement of membrane-associated cytokines. Arthritis Rheum 41: 1748-1759, 1998.

12. China Pharmacopoeia Committee: Chinese Pharmacopoeia. Chemical Industry Press, Beijing, China, 2010 (In Chinese).

13. Zhang YH, Song YJ and Jia Y: Observation of curative effect on the treatment of acute gouty arthritis with SM in 45 cases. Yunnan Zhong Yi Zhong Yao Za Zhi 24(4): 5-6, 2003 (In Chinese)

14. Liang GX and Duan JM: The curative effect observation on 68 cases of rheumatoid arthritis treated with Si long San Miao Formula. Guangming Journal of Chinese Medicine 22: 86-87, 2007 (In Chinese).

15. Guidance for Industry: Estimating the maximum safe starting dose in initial clinical trials for therapeutics in adult healthy volunteers. US Department of Health and Human Services, Food and Drug Administration, Center for Drug Evaluation and Research (CDER), Rockville, MD, 2005.

16. Hayami T, Pickarski M, Wesolowski GA, et al: The role of subchondral bone remodeling in osteoarthritis: reduction of cartilage degeneration and prevention of osteophyte formation by alendronate in the rat anterior cruciate ligament transection model. Arthritis Rheum 50: 1193-1206, 2004.

17. Gruber HE, Marshall GJ, Nolasco LM, Kirchen ME and Rimoin DL: Alkaline and acid phosphatase demonstration in human bone and cartilage: effects of fixation interval and methacrylate embedments. Stain Technol 63: 299-306, 1988.

18. Mankin HJ, Dorfman H, Lippiello L and Zarins A: Biochemical and metabolic abnormalities in articular cartilage from osteo-arthritic human hips. II. Correlation of morphology with biochemical and metabolic data. J Bone Joint Surg Am 53: 523-537, 1971.

19. YoshimiT,KikuchiT,ObaraT,etal:Effectsofhigh-molecular-weight sodium hyaluronate on experimental osteoarthrosis induced by the resection of rabbit anterior cruciate ligament. Clin Orthop Relat Res: 296-304, 1994.
20. Kim KM, Kim JM, Yoo YH, Kim JI and Park YC: Cilostazol induces cellular senescence and confers resistance to etoposide-induced apoptosis in articular chondrocytes. Int J Mol Med 29: 619-624, 2012.

21. Xu Y, Zhang ZJ, Geng F, et al: Treatment with Qing'E, a kidney-invigorating Chinese herbal formula, antagonizes the estrogen decline in ovariectomized mice. Rejuvenation Res 13: 479-488, 2010.

22. Pfaffl MW: A new mathematical model for relative quantification in real-time RT-PCR. Nucleic Acids Res 29: e45, 2001.

23. Kühn K, D'Lima DD, Hashimoto S and Lotz M: Cell death in cartilage. Osteoarthritis Cartilage 12: 1-16, 2004.

24. Jubb RW and Fell HB: The breakdown of collagen by chondrocytes. J Pathol 130: 159-167, 1980.

25. Rosenberg AE: Bones, joints, and soft tissue tumors. In: Robbins Pathologic Basis of Disease. Cotran RS, Kumar C and Collins T (eds). 6th edition. Philadelphia, PA, WB Saunders, p1253, 1999.

26. Naito K, Watari T, Muta T, et al: Low-intensity pulsed ultrasound (LIPUS) increases the articular cartilage type II collagen in a rat osteoarthritis model. J Orthop Res 28: 361-369, 2010.

27. Goldring MB, Otero M, Plumb DA, et al: Roles of inflammatory and anabolic cytokines in cartilage metabolism: signals and multiple effectors converge upon MMP-13 regulation in osteoarthritis. Eur Cell Mater 21: 202-220, 2011.

28. Wetzel M, Li L, Harms KM, et al: Tissue inhibitor of metalloproteinases-3 facilitates Fas-mediated neuronal cell death following mild ischemia. Cell Death Differ 15: 143-151, 2008.

29. Hu PF, Chen WP, Tang JL, Bao JP and Wu LD: Protective effects of berberine in an experimental rat osteoarthritis model. Phytother Res 25: 878-885, 2011.

30. Hashimoto S, Nishiyama T, Hayashi S, et al: Role of p53 in human chondrocyte apoptosis in response to shear strain. Arthritis Rheum 60: 2340-2349, 2009.

31. Del Carlo M Jr and Loeser RF: Nitric oxide-mediated chondrocyte cell death requires the generation of additional reactive oxygen species. Arthritis Rheum 46: 394-403, 2002.

32. Sun B, Lv L, Lu ZX and Yang SY: Study on drug-guide effect of Achyranthes bidentata in Sanmiao pill in arthritic rats. Zhongguo Zhong Yao Za Zhi 33: 2946-2949, 2008 (In Chinese).

33. Xiao HB, Sun ZL, Zhang HB and Zhang DS: Berberine inhibits dyslipidemia in C57BL/6 mice with lipopolysaccharide induced inflammation. Pharmacol Rep 64: 889-895, 2012.

34. Seo MJ, Kim SJ, Kang TH, et al: The regulatory mechanism of $\beta$-eudesmol is through the suppression of caspase- 1 activation in mast cell-mediated inflammatory response. Immunopharmacol Immunotoxicol 33: 178-185, 2011.

35. Shuler FD, Georgescu HI, Niyibizi C, et al: Increased matrix synthesis following adenoviral transfer of a transforming growth factor beta1 gene into articular chondrocytes. J Orthop Res 18: 585-592, 2000

36. Aigner T, Soeder S and Haag J: IL-1beta and BMPs - interactive players of cartilage matrix degradation and regeneration. Eur Cell Mater 12: 49-56, 2006 